\title{
AVALIAÇÃO DA FUNÇÃO HEPÁTICA EM PACIENTES SUBMETIDOS À ARTROPLASTIA TOTAL DO QUADRIL EM USO DE ENOXAPARINA
}

\author{
EVALUATION OF HEPATIC FUNCTION IN PATIENTS UNDERGOING TOTAL HIP \\ ARTHROPLASTY USING ENOXAPARIN
}

Felipe Vitiello Wink' ${ }^{1}$ Carlos Roberto Schwartsmann²

\section{RESUMO}

Objetivo: Avaliar as alterações hepáticas decorrentes do uso de enoxaparina para profilaxia da trombose venosa profunda em pacientes submetidos à artroplastia total do quadril. Métodos: Trinta e dois pacientes submetidos à artroplastia total do quadril, em caráter eletivo, utilizando enoxaparina, foram acompanhados por 65 dias com dosagens seriadas das enzimas hepáticas. Resultados: Foram encontradas alterações laboratoriais em até $75 \%$ dos pacientes durante o estudo, que normalizaram após a suspensão do tratamento. Não houve manifestação clínica de lesão hepática. Conclusão: As enzimas hepáticas elevam-se na maioria dos pacientes em uso de enoxaparina, sem correlação clínica, e normalizam após a suspensão do tratamento.

Descritores - Enoxaparina; Artroplastia de quadril; Insuficiência hepática; Trombose venosa

\section{ABSTRACT}

Objective: To evaluate hepatic changes due to the use of enoxaparin for prophylaxis of deep vein thrombosis in patients submitted to total hip arthroplasty. Methods: Thirty two patients submitted to elective total hip arthroplasty, using enoxaparin, were followed up for 65 days with serial doses of hepatic enzymes. Results: Laboratory changes were found in up to $75 \%$ of patients during the study, which normalized after suspension of the treatment. No clinical evidence of hepatic lesion was found. Conclusion: Increase in hepatic enzymes levels occurs in most patients using enoxaparin, but without clinical relevance, and normalizes after suspension of the treatment.

Keywords - Enoxaparin; Arthroplasty, replacement, hip; Hepatic insufficiency; Venous thrombosis

\section{INTRODUÇÃO}

Os fenômenos tromboembólicos relacionados ao pósoperatório de cirurgias ortopédicas são bem conhecidos, estudados, e representam altos custos médico-hospitalares. Há décadas estudam-se medicações capazes de reduzir o risco de trombose venosa profunda (TVP), e sua principal complicação, o tromboembolismo pulmonar $(\mathrm{TEP})^{(1)}$.

Diversos fármacos são empregados no intuito de prevenir e tratar esses fenômenos. Dentre eles encontramse a heparina não fracionada, a heparina de baixo peso molecular, como a enoxaparina, cumarínicos e novas medicações inibidoras seletivas do fator $\mathrm{Xa}^{(2)}$.
A eficácia anticoagulante e a segurança quanto ao risco hemorrágico apresentam bom embasamento na literatura médica, comparando-se os diversos fármacos entre $\mathrm{si}^{(3-6)}$; porém sua segurança quanto às alterações na função hepática não é bem conhecida ${ }^{(7-8)}$.

O objetivo deste trabalho é avaliar as alterações na função hepática de pacientes submetidos à artroplastia total do quadril em uso de enoxaparina.

\section{MÉTODOS}

Foram selecionados 32 pacientes, sendo 18 masculinos e 14 femininos, candidatos a artroplastia total do quadril, que preenchiam os seguintes critérios de inclusão: a) 18

1 - Médico Residente do Serviço de Ortopedia e Traumatologia do Complexo Hospitalar Santa Casa de Porto Alegre, RS, Brasil.

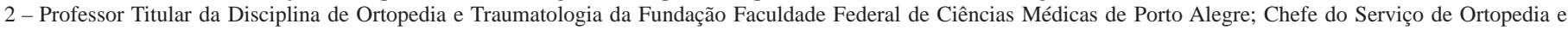
Traumatologia do Complexo Hospitalar Santa Casa de Porto Alegre, RS, Brasil. 
anos de idade ou mais; b) indicação de artroplastia total do quadril eletiva; c) pacientes que assinaram termo de consentimento, pelos seguintes motivos: $22(68,75 \%)$ por osteoartrose; quatro $(12,5 \%)$ por necrose avascular da cabeça femoral; quatro $(12,5 \%)$ por sequela de fratura do fêmur proximal; dois $(6,25 \%)$ por sequela de luxação congênita do quadril. Os critérios de exclusão foram: a) programados para cirurgia eletiva de prótese de quadril bilateral; b) com sangramento ativo ou alto risco de sangramento, contraindicando o tratamento com heparina de baixo peso molecular; c) portadores de contraindicação relacionada à droga a ser usada ou condição que impedisse o tratamento com anticoagulante; d) portadores de condição que contraindicasse a realização de flebografia bilateral; e) grávida ou lactante; f) mulheres em idade fértil que não estivessem utilizando métodos anticoncepcionais adequados; g) viciados em drogas ou álcool; h) em uso concomitante de inibidores da protease do HIV; i) em vigência de terapia com outro produto em estudo dentro do período de 30 dias antes do início da pesquisa clínica; j) em uso de compressão pneumática intermitente durante o período de tratamento ativo; k) submetidos concomitantemente a outra pesquisa clínica ou estudo; l) em terapia com outro tipo de anticoagulante que não pode ser interrompida ou outra medicação não permitida; $m$ ) doença hepática importante.

A média de idade era de 60 anos $( \pm 14,6)$, tendo o mais jovem 20 anos e o mais velho 82 anos, nenhum paciente apresentava doença hepática prévia, nem fazia uso de medicações que alterassem as provas de função hepática.

Os pacientes seguiram um protocolo uniforme de internação hospitalar no dia anterior a cirurgia, avaliação clínica e laboratorial pré-operatória conforme indicação médica, e coleta de amostras para dosagem das enzimas hepáticas aspartato aminotransferase (AST), alanina aminotransferase (ALT), gama-glutamil transpeptidase (GGT), fosfatase alcalina (FA), desidrogenase lática (DHL) e bilirrubinas total e direta (BT e BD).

O uso de enoxaparina foi iniciado 12 horas antes da cirurgia, com dose de 40mg subcutâneo, uma vez ao dia e mantido durante um período de 35 dias. Foram seriados os marcadores de função hepática após a cirurgia, e no sexto, décimo terceiro, trigésimo sexto e sexagésimo quinto dia pós-operatório.

Utilizou-se como valores de referência para AST 0-41U/L, ALT 0-45U/L, GGT 2-65U/L, FA 30-125U/L, DHL 100-220U/L, BT 0,1-1,2mg/dL e BD 0-0,4mg/dL, estabelecidos pelo laboratório Clinical Reference Laboratory, 8433 Quivira Road, Lenexa, Kansas, USA, onde foram analisadas as amostras dos pacientes.

\section{RESULTADOS}

Em quatro (13\%) pacientes encontrou-se uma ou mais enzimas elevadas no pré-operatório ALT e GGT. Estes casos foram excluídos do estudo. No pós-operatório imediato houve elevação na AST em três (9\%), ALT em dois (6\%), GGT em três (9\%), DHL em 10 (31\%), totalizando 13 (41\%) pacientes com alterações; no sexto dia pósoperatório, encontrou-se elevação na AST em seis (19\%), ALT três (9\%), GGT 12 (38\%), FA três (9\%), DHL cinco (16\%), com 15 (47\%) pacientes com alterações. No $13^{\circ}$ dia encontrou-se elevação na AST em dois (6\%), ALT em quatro (13\%), GGT em 12 (38\%), FA em cinco (9\%), DHL em 17 (53\%), totalizando 24 (75\%) pacientes com alterações. No $36^{\circ}$ dia encontrou-se elevação na AST em dois (6\%), ALT em um (3\%), GGT em quatro (13\%), FA em um (3\%), DHL em sete (22\%), totalizando 10 (31\%) pacientes com alterações. No $65^{\circ}$ dia encontrou-se elevação na AST em um (3\%), ALT em três (9\%), GGT em cinco (16\%), FA em três (9\%), DHL normalizada, totalizando oito (25\%) pacientes com alterações (Tabela 1).

Tabela 1 - Elevações nas enzimas hepáticas

\begin{tabular}{c|c|c|c|c|c|c}
\hline Enzima & Dia 0 & Dia 1 & Dia 6 & Dia 13 & Dia 36 & Dia 65 \\
\hline AST & 0 & $3(9 \%)$ & $6(19 \%)$ & $2(6 \%)$ & $2(6 \%)$ & $1(3 \%)$ \\
\hline ALT & $3(9 \%)$ & $2(6 \%)$ & $3(9 \%)$ & $4(13 \%)$ & $1(3 \%)$ & $3(9 \%)$ \\
\hline GGT & $3(9 \%)$ & $3(9 \%)$ & $12(38 \%)$ & $12(38 \%)$ & $4(13 \%)$ & $5(16 \%)$ \\
\hline FA & 0 & 0 & $3(9 \%)$ & $3(9 \%)$ & $1(3 \%)$ & $3(9 \%)$ \\
\hline DHL & 0 & $10(31 \%)$ & $5(16 \%)$ & $17(53 \%)$ & $7(22 \%)$ & 0 \\
\hline Total & $4(13 \%)^{\star}$ & $13(41 \%)$ & $15(47 \%)$ & $24(75 \%)$ & $10(31 \%)$ & $8(25 \%)$ \\
\hline
\end{tabular}

AST: aspartato aminotransferase; ALT: alanina aminotransferase; GGT: gama-glutamil transpeptidase; FA: fosfatase alcalina; DHL: desidrogenase láctica

* Pacientes excluídos do estudo

A análise das bilirrubinas não demonstrou alterações em nenhum paciente, tanto pré-operatórias quanto durante os 65 dias de seguimento. Nenhum paciente apresentou sinais ou sintomas referentes às alterações na função hepática (Figura 1).

\section{DISCUSSÃO}

Sabe-se que a heparina não fracionada apresenta uma relação com elevação assintomática de transaminases. Não existe correlação entre essas alterações e evidências clínicas de lesão hepática. Nos relatos publicados na literatura, houve normalização das enzimas hepáticas após suspensão do tratamento com heparina não fracionada ${ }^{(7)}$. 


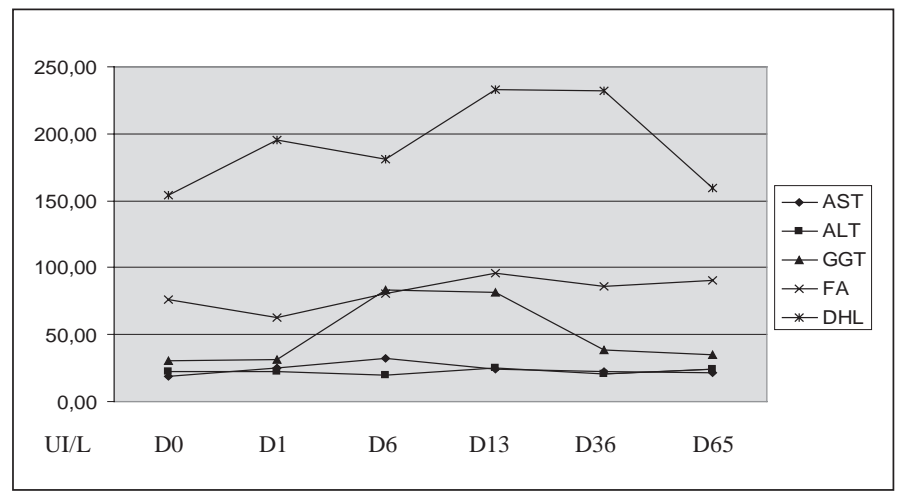

AST: aspartato aminotransferase; ALT: alanina aminotransferase; GGT: gama-glutamil transpeptidase; FA: fosfatase alcalina; DHL: desidrogenase láctica

Figura 1 - Curva média das enzimas hepáticas

Os recentes inibidores seletivos do fator Xa, ainda em fase clínica de estudo, apresentaram alterações significativas no valor das transaminases durante o tratamento, dose-dependentes, e sem alterações clinicamente evidentes. Os valores normalizaram-se após suspensão do tratamento ${ }^{(7-9)}$.

Em nosso estudo, encontramos alterações nas provas de função hepática na maioria dos pacientes tratados com enoxaparina, chegando a $75 \%$ no $13^{\circ}$ dia pós-operatório. Além das transaminases, elevaram-se as enzimas GGT, FA e DHL. O comportamento dessas alterações corresponde com o encontrado na literatura ${ }^{(3,6-8)}$, com elevação logo no início do tratamento, pico em torno de duas semanas de uso da medicação, e normalização após 60 dias.

\section{REFERÊNCIAS}

1. Deitelzweig SB, McKean SC, Amin AN, Brotman DJ, Jaffer AK, Spyropoulos AC. Prevention of venous thromboembolism in the orthopedic surgery patient. Cleve Clin J Med. 2008;75(Suppl 3):S27-36.

2. Dorr LD, Gendelman V, Maheshwari AV, Boutary M, Wan Z, Long WT. Multimodal thromboprophylaxis for total hip and knee arthroplasty based on risk assessment. J Bone Joint Surg Am. 2007;89(12):2648-57.

3. Christiansen HM, Lassen MR, Borris LC, Sørensen JV, Rahr HB, Jorgensen PW, et al. Biologic tolerance of two different low molecular weight heparins. Semin Thromb Hemost. 1991;17(4):450-4.

4. Senaran H, Acaroğlu E, Ozdemir HM, Atilla B. Enoxaparin and heparin comparison of deep vein thrombosis prophylaxis in total hip replacement patients. Arch Orthop Trauma Surg. 2006;126(1):1-5.

5. Leclerc JR, Geerts WH, Desjardins L, Jobin F, Laroche F, Delorme F, et al. Prevention of deep vein thrombosis after major knee surgery - a randomized,
Os valores de bilirrubina total e direta mantiveram-se dentro dos limites da normalidade durante todo o estudo. Não foram encontrados relatos na literatura de alterações no metabolismo das bilirrubinas em pacientes usuários de enoxaparina.

Apesar das alterações encontradas nesse estudo, nenhum paciente apresentou sinais ou sintomas clínicos relacionados às elevações das enzimas hepáticas. Essas alterações mostraram uma tendência de normalização após a suspensão do tratamento, com declínio gradativo dos valores durante o seguimento.

Esses dados se assemelham com os que encontramse na literatura ${ }^{(3,6-8)}$, e não foi possível associar nenhum desfecho desfavorável com a elevação das provas de função hepática em pacientes submetidos a artroplastia total do quadril, utilizando enoxaparina como profilaxia para TVP.

\section{CONCLUSÃO}

A maioria dos pacientes que utilizaram enoxaparina para profilaxia de TVP/TEP apresentaram alterações laboratoriais na função hepática, sem repercussão clínica. Essas alterações normalizaram com a suspensão do tratamento.

A maior alteração foi de DHL que, em 53\% dos casos, esteve elevada no $13^{\circ}$ dia de administração. double-blind trial comparing a low molecular weight heparin fragment (enoxaparin) to placebo. Thromb Haemost. 1992;67(4):417-23.

6. Jensen HP, Borris LC, Lassen MR, Sørensen JV, Rahr HB, Christiansen HM, et al. Low molecular heparin in prevention of thrombosis in orthopedic surgery. Ugeskr Laeger. 1993; 155(15):1109-15.

7. Carlson MK, Gleason PP, Sen S. Elevation of hepatic transaminases after enoxaparin use: case report and review of unfractionated and low-molecular-weight heparin-induced hepatotoxicity. Pharmacotherapy. 2001;21(1):108-13.

8. Harenberg J, Jörg I, Weiss C. Observations of alanine aminotransferase and aspartate aminotransferase in THRIVE studies treated orally with ximelagatran. Int J Toxicol. 2006;25(3):165-9.

9. Agnelli G, Eriksson BI, Cohen AT, Bergqvist D, Dahl OE, Lassen MR, et al. Safety assessment of new antithrombotic agents: Lessons from the EXTEND study on ximelagatran. Thromb Res. 2009;123(3):488-97. 